\title{
TRES NUEVAS ESPECIES DE COMMELINA (COMMELINACEAE) DEL CENTRO DE MÉXICO
}

\author{
Ana Rosa López-Ferrari, Adolfo Espejo-Serna y Jacqueline Ceja-Romero \\ Universidad Autónoma Metropolitana-Iztapalapa, División de Ciencias Biológicas \\ y de la Salud, Departamento de Biología, Herbario Metropolitano, \\ Apdo. postal 55-535, 09340 México, D.F. México. aes@xanum.uam.mx
}

\section{RESUMEN}

En este trabajo se describen como nuevas e ilustran Commelina congestispatha López-Ferrari, Espejo et Ceja, Commelina ramosissima López-Ferrari, Espejo et Ceja y Commelina queretarensis López-Ferrari, Espejo et Ceja. La primera es conocida de los estados de Michoacán, Guanajuato y Querétaro, la segunda proviene de los estados de Guanajuato, Querétaro y Michoacán y la tercera se conoce sólo del estado de Querétaro, de los municipios de San Joaquín y Landa de Matamoros.

Palabras clave: centro de México, Commelina, Commelinaceae.

\begin{abstract}
Commelina congestispatha López-Ferrari, Espejo et Ceja, Commelina ramosissima López-Ferrari, Espejo et Ceja, and Commelina queretarensis López-Ferrari, Espejo et Ceja, are described and illustrated as new in this paper. The former is known from the states of Michoacán, Guanajuato and Querétaro, the second was collected in the states of Guanajuato, Querétaro and Michoacán, and the last one comes from Querétaro in the municipios of San Joaquín and Landa de Matamoros.
\end{abstract}

Key words: central Mexico, Commelina, Commelinaceae.

\section{INTRODUCCIÓN}

El género Commelina tiene entre 170 (Faden y Hunt, 1991) y 250 especies (Tucker, 1989) de distribución cosmopolita, con el mayor número de representantes 
en el Viejo Mundo (Dahlgren et al., 1985). Matuda (1956 a y b) citó para México nueve especies, a su vez Espejo y López-Ferrari, en 1995, registraron 17 taxa y posteriormente se describieron dos más (López-Ferrari et al., 1997), por lo cual actualmente para el país se tiene registrado un total de 19 taxa. Sin embargo, con el incremento de las exploraciones botánicas es probable que esta cifra crezca aún más en el futuro.

Como resultado del trabajo de recolección y de la revisión de material herborizado, con el fin de completar el fascículo de las Commelinaceae para la Flora del Bajío y de Regiones Adyacentes, encontramos tres novedades del género Commeli$n a$, por lo que proponemos:

Commelina congestispatha López-Ferrari, Espejo et Ceja, sp. nov. Fig. 1

Herba erecta usque $60 \mathrm{~cm}$ alta, radicibus tenuibus cylindricis. Folia ovata, ovato-lanceolata vel elliptica, $4-9 \mathrm{~cm}$ longa, $1.5-3.6 \mathrm{~cm}$ lata. Inflorescentiae plures terminales, 3-10 catervatim aggregatae et cum dua folia affines, pedunculis nullis vel minus quam $1 \mathrm{~cm}$ longis, bracteis spathaceis depresso-ovatis, in extremo posteriore connatis, carinatis et cordatis ubi extensis, dense pubescentibus; petala 2 azurea pallida, unguide 1-1.5 mm longo. Capsula hemiglobosa triquetra $3 \mathrm{~mm}$ longa.

Planta herbácea perenne, erecta en la base, a veces decumbente en la porción superior, de 25 a $60 \mathrm{~cm}$ de alto; raíces delgadas, cilíndricas, de hasta $18 \mathrm{~cm}$ de largo; tallos rectos, generalmente ramificados desde la base; hojas ovadas, ovado-lanceoladas o elípticas, las vainas laxas, de 1.2 a $1.5 \mathrm{~cm}$ de largo, cortamente pubescentes, ciliadas en los márgenes, láminas de $4 \mathrm{a} 9 \mathrm{~cm}$ de largo, de 1.5 a $3.6 \mathrm{~cm}$ de ancho, agudas a acuminadas, atenuadas y algo oblicuas en la base, corta y esparcidamente pubescentes en ambas superficies; inflorescencias terminales, agrupadas en el ápice de los tallos en conjuntos de 3 a 10, sésiles a semisésiles, los pedúnculos, cuando presentes, de menos de $1 \mathrm{~cm}$ de largo; brácteas espatáceas deprimido-ovadas, carinadas y cordadas cuando extendidas, connadas en su extremo posterior, de 1.8 a 2.1 $\mathrm{cm}$ de largo, de ca. $3 \mathrm{~cm}$ de ancho, agudas a apiculadas en el ápice, truncadas en el extremo opuesto, densamente pubescentes, particularmente hacia la base, conspicuamente nervadas; flores de ca. $1.5 \mathrm{~cm}$ de diámetro; sépalos tres, los dos inferiores oblongos, connados en su mitad inferior, de 3.7 a $4 \mathrm{~mm}$ de largo, de ca. $2 \mathrm{~mm}$ de ancho, hialinos, el superior triangular, de ca. $2.7 \mathrm{~mm}$ de largo, de ca. $1.7 \mathrm{~mm}$ de ancho, verde a hialino; pétalos dos, iguales, suborbiculares a reniformes, de 6.7 a $7.2 \mathrm{~mm}$ de largo, de 7.7 a $8.7 \mathrm{~mm}$ de ancho, de color azul pálido, la uña de 1 a $1.5 \mathrm{~mm}$ de largo; 

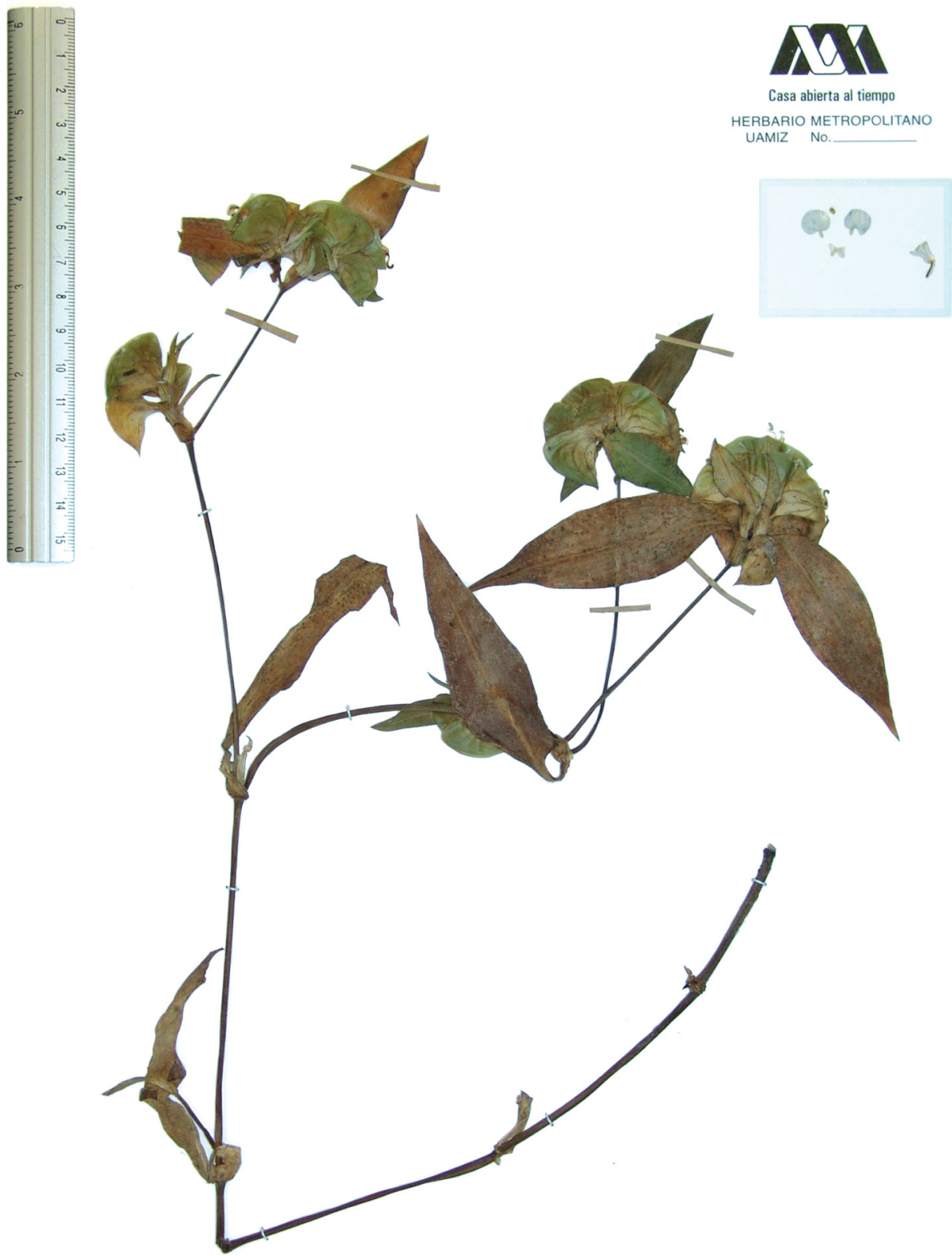

Fig. 1. Commelina congestispatha López-Ferrari, Espejo et Ceja (A. Espejo et al. 6903). 
estambres fértiles tres, los dos laterales con los filamentos de ca. $4.7 \mathrm{~mm}$ de largo y con las anteras oblongas, de ca. $2 \mathrm{~mm}$ de largo, el medio con el filamento de ca. 4.7 $\mathrm{mm}$ de largo y la antera sagitada de ca. $2.5 \mathrm{~mm}$ de largo, estaminodios cruciformes, amarillos, de $1 \mathrm{~mm}$ de largo; ovario semigloboso, de ca. $2 \mathrm{~mm}$ de largo, de ca. 1.5 $\mathrm{mm}$ de diámetro, verde; estilo filiforme, de $6.5 \mathrm{~mm}$ de largo, blanco, estigma capitado, azul; cápsula semiglobosa, triquetra, de ca. $3 \mathrm{~mm}$ de largo, de ca. $4.5 \mathrm{~mm}$ de diámetro, algo rugosa; semillas globosas a semiglobosas, lisas, umbilicadas, de ca. $2 \mathrm{~mm}$ de diámetro, pardas rojizas.

Tipo: Guanajuato, municipio de Cuerámaro $1.2 \mathrm{~km}$ después de Cañada de Corralejo, rumbo a la barranca de El Chilar, 20³4'41" N; 101²4'51" W, 1900 m s.n.m., talud en bosque tropical caducifolio, 4.IX.2006, A. Espejo, A. López-Ferrari, J. Ceja y J. García Cruz 6903 (fotografía digital y tarjeta con disección floral) (holotipo: UAMIZ; isotipos: CIIDIR, IBUG, IEB, QMEX, UJAT).

Paratipos: Guanajuato, municipio de Apaseo el Grande, cerca de Ixtla, 1900 m s.n.m., bosque tropical caducifolio perturbado, 16.VIII.1986, J. Rzedowski 40348 (IEB); municipio de Pénjamo, $39 \mathrm{~km}$ al SO de Cuerámaro, sobre el camino a la Barrranca del Chilar, 1900 m s.n.m., bosque tropical caducifolio, 4.X.1988, J. Rzedowski 47216 (IEB); municipio de Tarimoro, $6.7 \mathrm{~km}$ después de Tarimoro, rumbo a La Cuesta, 20¹9'32" N, 10043'57" W, 2026 m s.n.m., selva baja caducifolia perturbada, 5.IX.2006, J. Ceja et al. 1776 (IEB, UAMIZ). QuerétARO, municipio de Landa, ca. 5 km después de Zoyapilca, rumbo a Soledad de Guadalupe, 2121'08" N, 99¹6'42" W, 1330 m s.n.m., bosque de encino, 8.IX.2006, J. Ceja et al. 1791 (IEB, UAMIZ); municipio de El Marqués, camino arriba de La Cañada (Heidi’s place), 1990 m s.n.m., matorral, 18.IX.1988, E. Argüelles 3142 (IEB). MichOACÁN, municipio de Susupuato, La Ziranda, $1650 \mathrm{~m}$ s.n.m., vegetación secundaria derivada del bosque tropical caducifolio, 26.IX.1989, J. Rzedowski 49019 (IEB); municipio de Tlazazalca, presa La Yerbabuena, $1950 \mathrm{~m}$ s.n.m., pastizal húmedo a la orilla de la presa, 8.VIII.1990, E. Pérez y E. García 1596 (IEB, MEXU, UAMIZ).

C. congestispatha debe su nombre a que las inflorescencias se agrupan en el ápice de los tallos en número de tres o más (Fig. 1).

El nuevo taxon podría confundirse, particularmente en material herborizado, con $C$. erecta L., ya que ambas presentan las brácteas espatáceas connadas en su extremo posterior y flores con dos pétalos. Sin embargo, en $C$. congestispatha las inflorescencias siempre se agrupan en número de tres o más en el ápice de los tallos 
y están asociadas a dos hojas, mientras que en $C$. erecta éstas pueden ser terminales y/o axilares, generalmente son solitarias, y en ocasiones se presentan en grupos de dos o máximo tres. Otra diferencia entre ambas especies es el tamaño de las flores, que en $C$. congestispatha son más pequeñas, con los pétalos de 6.7-7.2 $\mathrm{mm}$ de largo y cortamente unguiculados, mientras que en $C$. erecta son de 11-19 mm y están largamente unguiculados.

Commelina congestispatha se ha registrado de bosques tropicales caducifolios, así como de bosques de encino, creciendo más bien en lugares perturbados entre los 1300 y los 2050 m s.n.m. Florece de agosto a octubre.

Commelina ramosissima López-Ferrari, Espejo et Ceja, sp. nov. Fig. 2

Herba erecta usque $80 \mathrm{~cm}$ alta, supra valde ramosa, radicibus tuberosis distaliter incrassatis. Folia lanceolata vel anguste lanceolata, 8-14 cm longa, 8-14 mm lata. Inflorescentiae solitariae axillares et terminales, pedunculis dense pubescentibus 2.5$10 \mathrm{~cm}$ longis, angulo inter pedunculum et bracteas spathaceas majore quam $90^{\circ}$, bracteis spathaceis in extremo posteriore liberis, acuminatis vel longe acuminatis, late ovatis et cordatis ubi extensis, sparse pubescentibus; petala 3 aequalia vel subaequalia, azurea, unguide ca. $3 \mathrm{~mm}$ longo. Capsula ellipsoidalis triquetra 6-6.4 $\mathrm{mm}$ longa.

Planta herbácea perenne, erecta, de hasta $80 \mathrm{~cm}$ de alto; raíces tuberosas, engrosadas en su parte distal; tallos rectos, densamente pilosos, muy ramificados en la porción superior, las ramas ascendentes a difusas; hojas lanceoladas a angostamente elíptico-lanceoladas, las vainas laxas, algo escariosas, con frecuencia teñidas de púrpura, de $1 \mathrm{a} 3 \mathrm{~cm}$ de largo, densamente pilosas, ciliadas en la parte superior, láminas de 8 a $14 \mathrm{~cm}$ de largo, de 8 a $14 \mathrm{~mm}$ de ancho, agudas a acuminadas, la base atenuada, de color verde pálido, las inferiores esparcidamente pilosas a glabrescentes en la haz, esparcidamente pilosas en el envés, las superiores densamente pilosas en ambas superficies; inflorescencias varias a numerosas, solitarias, axilares y terminales, pedúnculos densamente pubescentes, de 2.5 a $10 \mathrm{~cm}$ de largo, el ángulo entre los pedúnculos y las brácteas espatáceas mayor de 90 grados; brácteas espatáceas libres en su extremo posterior, acuminadas a largamente acuminadas, ampliamente ovadas y cordadas cuando extendidas, de 2.7 a $4.3 \mathrm{~cm}$ de largo, de 2 a $3 \mathrm{~cm}$ de ancho, con frecuencia teñidas de púrpura, esparcidamente pubescentes por fuera, esparcidamente vellosas por dentro, conspicuamente nervadas; flores de ca. $2.5 \mathrm{~cm}$ de diámetro; sépalos tres, oblongos a oblongo-elípticos, de 5.8 a $6.4 \mathrm{~mm}$ de largo, de 3 a $3.2 \mathrm{~mm}$ de ancho; pétalos tres, iguales o subiguales, obovados, unguiculados, de ca. $11 \mathrm{~mm}$ de 
Acta Botanica Mexicana 87: 71-81 (2009)

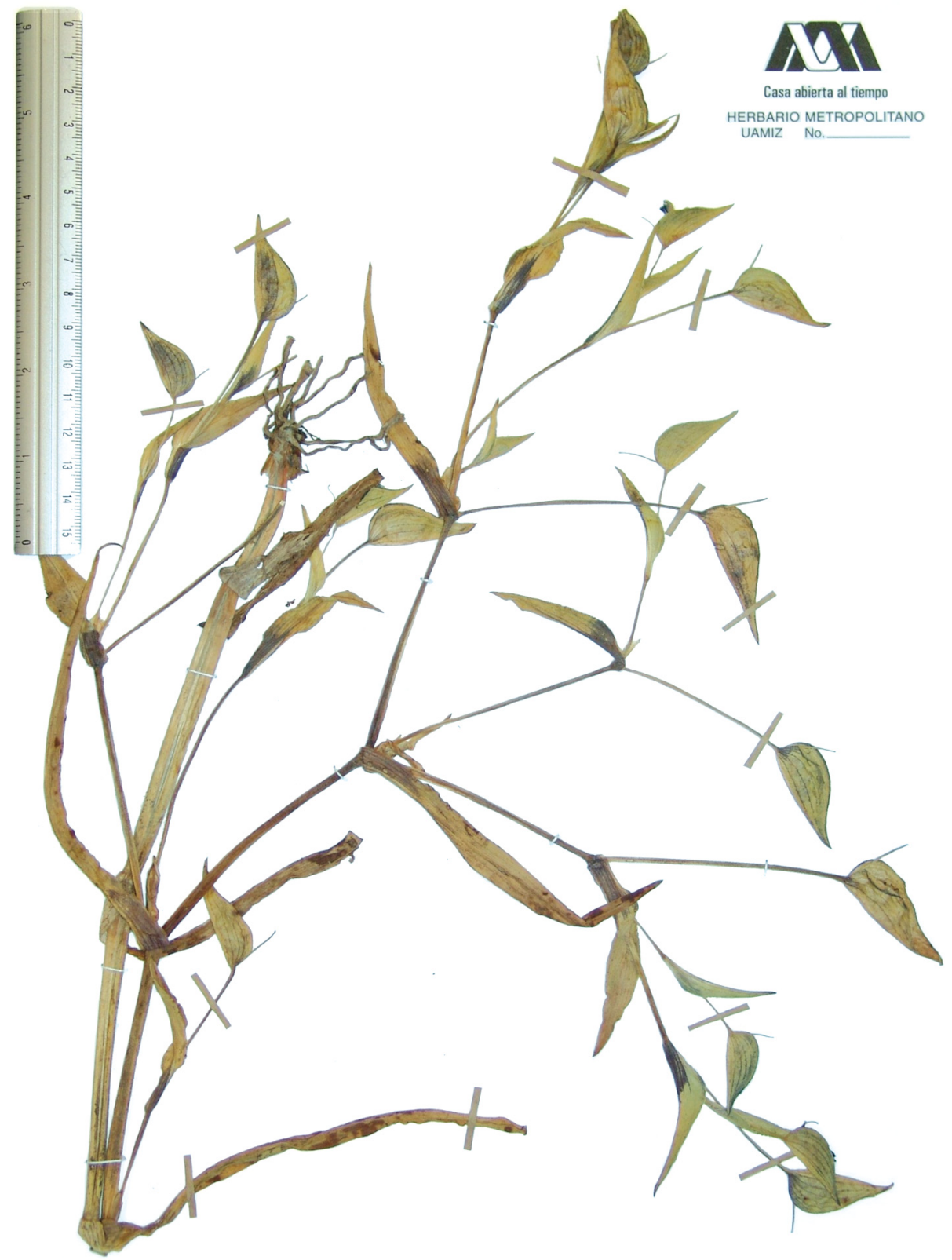

Fig. 2. Commelina ramosissima López-Ferrari, Espejo et Ceja (J. Rzedowski 47173). 
largo, de ca. $7.5 \mathrm{~mm}$ de ancho, de color azul, la uña de ca. $3 \mathrm{~mm}$ de largo; estambres fértiles tres, los dos laterales con los filamentos de ca. $7 \mathrm{~mm}$ de largo y con las anteras oblongas de 1.5 a $1.8 \mathrm{~mm}$ de largo, el medio con el filamento de ca. $5 \mathrm{~mm}$ de largo y la antera sagitada de ca. $2.2 \mathrm{~mm}$ de largo, estaminodios cruciformes, amarillos, de ca. $1.5 \mathrm{~mm}$ de largo; ovario ovoide, de ca. $2 \mathrm{~mm}$ de largo, de ca. $1 \mathrm{~mm}$ de diámetro, estilo de ca. $6 \mathrm{~mm}$ de largo, enroscado en el ápice; cápsula elipsoide, triquetra, de 6 a $6.4 \mathrm{~mm}$ de largo, de 4 a $5 \mathrm{~mm}$ de diámetro, cortamente apiculada; semillas elipsoides u oblongas, truncadas, foveoladas, de ca. $2.7 \mathrm{~mm}$ de largo, pardas oscuras.

Tipo: Guanajuato, municipio de Pénjamo, $22 \mathrm{~km}$ al SW de Cuerámaro, sobre el camino a la Barranca del Chilar, 2200 m s.n.m., orilla de un charco, 4.IX.1988, J. Rzedowski 47173 (holotipo: IEB; isotipos: IBUG, MEXU, UAMIZ).

Paratipos: Guanajuato, municipio de Pénjamo, $22 \mathrm{~km}$ al SW de Cuerámaro, sobre el camino a la Barranca del Chilar, 2200 m s.n.m., orilla de un charco, 4.X.1987, J. Rzedowski 44978 (IEB, UAMIZ); municipio de Pénjamo, 24 km al SW de Cuerámaro, sobre el camino a la Barranca del Chilar, 2200 m s.n.m., orilla de arroyo, 4.IX.1988, J. Rzedowski 47180 (IEB, UAMIZ). QuerétARO, municipio de Colón, $3 \mathrm{~km}$ al SE de Trigos, sobre el camino a Colón, 2500 m s.n.m., pastizal con arbustos, 27.VIII.1989, J. Rzedowski 48801 (IEB); MichOACÁN, municipio de Tlazazalca, presa La Yerbabuena, pastizal húmedo, orilla de la presa, 8.VIII.1990, E. Pérez y E. García L. 1598 (IEB, UAMIZ); municipio de Villa Jiménez, $15 \mathrm{~km}$ al N de Villa Jiménez, sobre la carretera a Puruándiro, 1750 m s.n.m., terrenos encharcados a la orilla del camino, 3.IX.1989, J. Rzedowski 48843 (IEB).

El nombre de la especie hace referencia a las plantas generalmente muy ramificadas en la porción superior de los tallos.

Commelina ramosissima es escasa en el área de estudio, en donde habita a la orilla de cuerpos de agua, entre los 2200 y 2500 m s.n.m. Florece de septiembre a octubre. La presencia de los tallos ramificados en la porción superior, aunada a las brácteas espatáceas, que forman un ángulo mayor de $90^{\circ}$ con los pedúnculos, que son largos y densamente pubescentes, caracterizan a C. ramosissima. El nuevo taxon se asemeja, a primera vista, a $C$. pallida Willd., ya que ambas especies tienen tallos ramificados y pedúnculos alargados, aunque en $C$. pallida las inflorescencias se presentan en número de 2 a 4 por nudo y las flores son pequeñas, de ca. $1 \mathrm{~cm}$ de diámetro, mientras que en C. ramosissima las inflorescencias siempre son solitarias y las flores miden $2.5 \mathrm{~cm}$ de diámetro. 
Commelina queretarensis López-Ferrari, Espejo et Ceja, sp. nov. Fig. 3

Herba decumbens vel erecta usque $1 \mathrm{~m}$ longa. Folia longissime elliptica ad lineari-lanceolata, 7-17 cm longa, 8-13 mm lata. Inflorescentiae solitariae axillares et terminales, pedunculis breviter sparseque pubescentibus, $1.8-4.5 \mathrm{~cm}$ longis, bracteis spathaceis in extremo posteriore liberis, acutis ad acuminatis, late ovatis et cordatis ubi extensis, sparse pubescentibus et cum nervo inferiore pilis longis multiseriatis per totam longitudinem instructo; petala 3 subaequalia azurea, unguide 8-9 $\mathrm{mm}$ longo. Capsula ignota.

Planta herbácea perenne, erecta a decumbente, de hasta $1 \mathrm{~m}$ de alto, raíces tuberosas; tallos rectos, corta y esparcidamente pubescentes, algo ramificados en la porción superior; hojas muy largamente elípticas a linear-lanceoladas, las vainas laxas, algo escariosas, de 1.7 a $2.7 \mathrm{~cm}$ de largo, corta y esparcidamente pubescentes, ciliadas en la parte superior, láminas de 7 a $17 \mathrm{~cm}$ de largo, de 8 a $13 \mathrm{~mm}$ de ancho, agudas a acuminadas, la base atenuada, de color verde oscuro, glabras en ambas superficies, cortamente escabriúsculas en el margen; inflorescencias solitarias, axilares y terminales, pedúnculos corta y esparcidamente pubescentes, de 1.8 a $4.5 \mathrm{~cm}$ de largo, el ángulo entre los pedúnculos y las brácteas espatáceas de 90 grados o menos; brácteas espatáceas libres en su extremo posterior, agudas a acuminadas, ampliamente ovadas y cordadas cuando extendidas, de 2.5 a $4.1 \mathrm{~cm}$ de largo, de 2 a $3.4 \mathrm{~cm}$ de ancho, esparcidamente pubescentes por fuera, glabras por dentro, conspicuamente nervadas, la nervadura inferior con pelos largos, multiseriados, en toda su longitud; flores de ca. $2.5 \mathrm{~cm}$ de diámetro; sépalos tres, los inferiores suborbiculares, algo asimétricos, de 5.5 a $6 \mathrm{~mm}$ de largo, de ca. $5 \mathrm{~mm}$ de ancho, hialinos a azules; pétalos tres, subiguales, semicirculares a deltados, unguiculados, de 8 a $9 \mathrm{~mm}$ de largo, de ca. $13 \mathrm{~mm}$ de ancho, de color azul, la uña de ca. $3 \mathrm{~mm}$ de largo; estambres fértiles tres, los dos laterales con los filamentos de ca. $15 \mathrm{~mm}$ de largo y con las anteras oblongas de 2 a $2.5 \mathrm{~mm}$ de largo, el medio con el filamento de ca. $10 \mathrm{~mm}$ de largo y la antera sagitada de ca. $3 \mathrm{~mm}$ de largo, estaminodios cruciformes, amarillos, de 1.6 a $2 \mathrm{~mm}$ de largo; ovario angostamente ovoide, de 1.7 a $2 \mathrm{~mm}$ de largo, de ca. 1 $\mathrm{mm}$ de diámetro, estilo de ca. $15 \mathrm{~mm}$ de largo, cápsula no vista.

Tipo: QuerÉTARo: municipio de San Joaquín, Deconí, por el camino a Maravillas, $20^{\circ} 55^{\prime} 03^{\prime \prime} \mathrm{N}, 9^{\circ} 32^{\prime} 35^{\prime \prime} \mathrm{W}, 2370$ m s.n.m., laderas calizas con bosque de encinos y elementos de bosque mesófilo de montaña, 17.IX.2006, S. Zamudio y R. Alcalá 13578 (holotipo: IEB; isotipos: MEXU, UAMIZ). 
Acta Botanica Mexicana 87: 71-81 (2009)

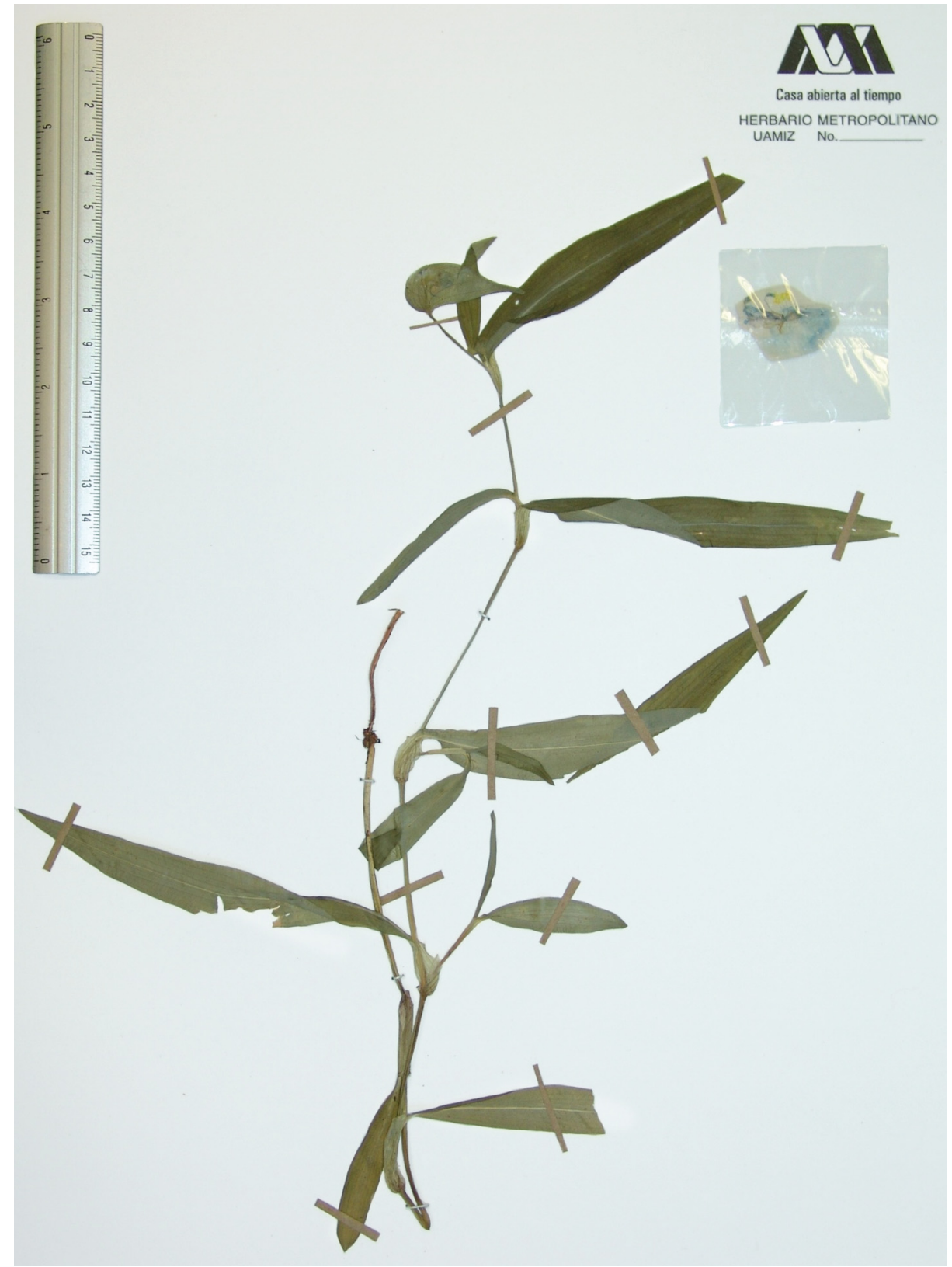

Fig. 3. Commelina queretarensis López-Ferrari, Espejo et Ceja (S. Zamudio y R. Alcalá 13578). 
Paratipos: Querétaro, municipio de Landa de Matamoros, El Banco, 1.5 $\mathrm{km}$ al sureste de La Yesca, $1820 \mathrm{~m}$ s.n.m., bosque de Quercus, Pinus y Cupressus, 25.VII.1989, H. Rubio 916 (IEB); municipio de Landa de Matamoros, $2 \mathrm{~km}$ al sureste de La Yesca, El Banco, 1850 m s.n.m., bosque de encino, cedro blanco y pino, 22.IX.1990, H. Rubio 1991 (IEB, UAMIZ).

Commelina queretarensis se conoce sólo del estado de Querétaro, de los municipios de San Joaquín y de Landa de Matamoros, de allí el nombre de la especie. Las plantas del nuevo taxon aquí propuesto son escasas en los sitios donde crecen. Habita en laderas calizas dentro de bosques de encino y de encino-pino-cedro, entre los 1820 y los 2370 m s.n.m. Florece de julio a septiembre.

La nueva especie presenta afinidades con Commelina dianthifolia Delile, sin embargo se distingue de ésta por presentar las hojas más anchas, no conduplicadas y de color verde oscuro y por sus brácteas espatáceas pubescentes y con una característica línea basal de pelos multiseriados.

\section{AGRADECIMIENTOS}

Queremos agradecer a los DD. Jerzy Rzedowski, Graciela Calderón y Eleazar Carranza la revisión crítica del manuscrito así como sus valiosas sugerencias y observaciones al mismo. Al Dr. Rzedowski por elaborar la diagnosis latina de las nuevas especies. A Aniceto Mendoza Ruiz, colega de la Universidad Autónoma Metropolitana Iztapalapa, por su apoyo incondicional en el trabajo de campo. Finalmente agradecemos a los curadores de los herbarios consultados por las facilidades otorgadas y al Consejo Nacional de Ciencia y Tecnología por el apoyo económico brindado a los dos primeros autores para una estancia de trabajo en el Centro Regional del Bajío, Instituto de Ecología, A.C., durante febrero del 2008.

\section{LITERATURA CITADA}

Dahlgren, R. M. T., H. T. Clifford y P. Yeo. 1985. The families of the Monocotyledons. Springer-Verlag. Berlin. 520 pp.

Espejo, A. y A. R. López-Ferrari. 1995. Las monocotiledóneas mexicanas, una sinopsis florística. I. Lista de referencia. Parte 4. Commelinaceae, Convallariaceae, Costaceae, Cyclanthaceae y Cymodoceaceae. Consejo Nacional de la Flora de México, A.C., 
Universidad Autónoma Metropolitana y Comisión Nacional para el Conocimiento y Uso de la Biodiversidad. México, D.F. 53 pp.

Faden, R. B. y D. R. Hunt. 1991. The classification of Commelinaceae. Taxon 40: 19-31.

López-Ferrari, A. R., A. Espejo y J. Ceja. 1997. Dos nuevas especies de Commelina (Commelinaceae) mexicanas. Acta Bot. Mex. 41: 9-16.

Matuda, E. 1956a. Las commelináceas del Estado de México. Dirección de Recursos Naturales del Gobierno del Estado de México. Toluca, México. 46 pp.

Matuda, E. 1956b. Las commelináceas mexicanas. Anales Inst. Biol. Mex. 26(2): 303-340. Tucker, G. C. 1989. The genera of Commelinaceae in the southeastern United States. J. Arnold Arbor. 70: 97-130. 
